\title{
The effect of magnetic interaction in barium hexaferrite particles
}

\author{
M. García del Muro, X. Batlle, and A. Labarta \\ Departament de Física Fonamental, Universitat de Barcelona, Diagonal 647, 08028 Barcelona, Spain
}

J. M. González and M. I. Montero

Instituto de Ciencia de Materiales de Madrid (C.S.I.C.), Cantoblanco, 28049 Madrid, Spain

\begin{abstract}
The effect of interactions on the magnetic relaxation of nanocrystalline hexagonal barium hexaferrite $\mathrm{BaFe}_{10.4} \mathrm{Co}_{0.8} \mathrm{Ti}_{0.8} \mathrm{O}_{19}$ is discussed. We had previously shown that according to the $T \ln \left(t / \tau_{0}\right)$ scaling, an enhancement of the lowest-energy barriers was detected when demagnetizing interactions were dominant. Also, the Henkel plots obtained for particles of about $10 \mathrm{~nm}$ of mean diameter showed that the overall interactions were demagnetizing. In the present work, we have modified the interactions by milling the particles with a nanosized $\mathrm{SiO}_{2}$ powder. Dipolar interactions are modified by breaking the particle aggregates. The observed overall interactions resulted to be also demagnetizing for the milled sample. The time dependence of the magnetization was analyzed according to two different procedures: the fluctuation field and activation volume analysis and the $T \ln \left(t / \tau_{0}\right)$ scaling. Activation volumes were found to increase with demagnetizing interactions and the leading demagnetizing mechanism appeared to shift from an individual particle mode for the unmilled sample to a collective one for the milled sample. The second approach showed larger relaxation rates at short times for the milled sample. The effective energy barrier distribution obtained from the scaling suggested that demagnetizing interactions increased in the milled sample, which led to an enhancement of the amount of the lowest-energy barriers. (C) 1997 American Institute of Physics. [S0021-8979(97)57608-0]
\end{abstract}

$M$-type barium hexaferrite has been widely studied because of its technological applicability. In the last years many efforts have been devoted to the development of synthesis procedures that leads to controlled-size particles, and to the knowledge of its basic properties. The study of the magnetic properties of barium hexaferrite nanoparticles showed that interparticle interactions can affect their magnetic behaviour. The selected system has previously shown demagnetizing interactions. ${ }^{1}$ In the present work, we have modified the interactions by milling the particles with a nanosized $\mathrm{SiO}_{2}$ powder. Dipolar interactions are modified by breaking the particle aggregates. According to the results from Ido et al. ${ }^{2}$ the resultant interaction will depend on the way the particles were grouped. Barium hexaferrite particles tend to pile up, leading to stacks, where interactions are magnetizing, although particle clusters are also observed, where interactions are demagnetizing. The effect of interactions on the magnetic relaxation has been analyzed in terms of fluctuation field and activation volume. To complete the analysis and the interpretation of the results, the $T \ln \left(t / \tau_{0}\right)$ scaling of the time dependence of the thermoremanence (which provides the energy barrier distribution) have been done.

Nanocrystalline $\mathrm{BaFe}_{10.4} \mathrm{Co}_{0.8} \mathrm{Ti}_{0.8} \mathrm{O}_{19}$ particles, with a mean particle diameter of about $10 \mathrm{~nm}$, were prepared by using the glass crystallization method. ${ }^{3}$ Two different samples were prepared compacting the nanocrystalline powder by cold pressing (in order to avoid particle rotations during magnetic measurements): sample 1 (S1) contains only barium hexaferrite powder, and sample 2 (S2) contains a mixture of barium hexaferrite powder and nanosized $\mathrm{SiO}_{2}$ with a mean particle diameter of about $3 \mathrm{~nm}$. The admixture was prepared by milling for $5 \mathrm{~min}$ the powders in a $60 \%$ volume fraction of silica. The milling was carried out by means of a planetary ball mill, using hardened stainless steel jars and balls in a weight of balls-to-weight of sample ratio of $40: 1$.

Magnetization measurements were carried out with a SQUID magnetometer in the temperature range 4.2-300 K and under magnetic fields of up to $50 \mathrm{kOe}$. Isothermal hysteresis loops were recorded at several temperatures to obtain the coercive force $\left(H_{c}\right)$. In order to obtain the fluctuation field, the time dependence of the magnetization was measured under several applied demagnetizing fields in the region of the demagnetization curve corresponding to the critical field, after zero-field cooling the sample from room temperature and saturating it in a $50 \mathrm{kOe}$ field at the measuring temperature. Time dependence of the thermoremanence in the temperature range $9-250 \mathrm{~K}$ was measured at zero field after field cooling the sample at 200 Oe from room temperature. These results were analyzed in terms of the $T \ln \left(t / \tau_{0}\right)$ scaling.

The hysteresis loops were recorded from 4 to $70 \mathrm{~K}$. The coercive field values as a function of temperature were fitted assuming that for an assembly of randomly oriented particles in the blocked state $H_{c}=H_{c}(0)\left(1-A T^{0.77}\right),{ }^{4}$ with $A=\left(\beta k_{B} /\langle E\rangle\right)^{0.77}$ and $\beta$ is given by the relation between the experimental measuring time, $t_{m}$, and the attempting characteristic time which governs the Arrhenius law for magnetic relaxation $\left[\beta=\ln \left(t_{m} / \tau_{0}\right)\right]$. Although $\tau_{0}$ is usually taken as $10^{-9} \mathrm{~s}$, in the present case we use $10^{-12}$ due to the occurrence of demagnetizing interaction effects (see below and Ref. 1), leading to $\beta \sim 32$ for SQUID measurements $\left(t_{m} \sim 50-100 \mathrm{~s}\right)$. The $T^{0.77}$ law is verified below $30 \mathrm{~K}$ for both samples. The coercive force at $0 \mathrm{~K}$ obtained from the fit are $H_{c}(0)=6388 \mathrm{Oe}(\mathrm{S} 1)$ and $H_{c}(0)=5950 \mathrm{Oe}(\mathrm{S} 2)$, and $H_{c}$ values for S2 always remain below S1 values. The mean anisotropy energy is very similar for the two samples: 


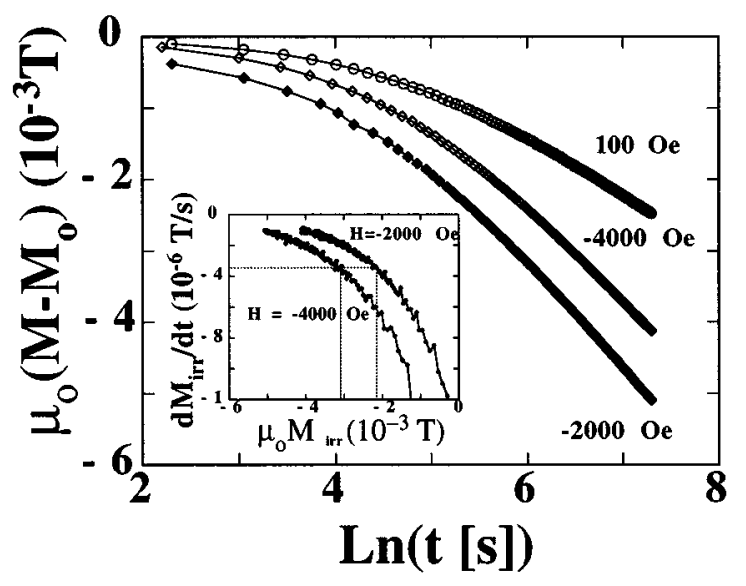

FIG. 1. The time variation of the magnetization measured at diverse inversion fields (where $M_{0}$ is the magnetization after fixing this field) applied after saturating the sample 1 at $43 \mathrm{~K}$. Inset: the $d \mu_{0} M / d t=c t$. condition applied to these curves to obtain the $\left(\mu_{0} M_{\text {irr }}, \mu_{0} H_{\text {int }}\right)$ values.

$\langle E\rangle=2.7 \times 10^{-13} \mathrm{erg}$ and $\langle E\rangle=2.8 \times 10^{-13} \mathrm{erg}$ for $\mathrm{S} 1$ and $\mathrm{S} 2$, respectively. According to these results, by mixing the ferrite nanoparticles with $\mathrm{SiO}_{2}$ the mean height of the energy barrier does not change, but S2 demagnetizes more easily. We then understand that in $\mathrm{S} 2$ demagnetizing interactions have been enhanced with respect to $\mathrm{S} 1$.

When studying the demagnetizing processes, the critical field $\left(H_{\mathrm{cr}}\right)$ is also a representative parameter. This is the field at which $d M / d H$ corresponding to the demagnetization branch of the hysteresis loop presents the maximum value, and expresses the field for which the maximum rate of magnetization inversion process occurs. In the present case, the obtained $H_{\mathrm{cr}}$ values at $4 \mathrm{~K}$ were about $20 \%$ smaller than the corresponding coercive field, and this difference decreases with temperature.

The experimental procedure used to determine the fluctuation field was proposed by Givord et al., ${ }^{5}$ and is compatible with the magnetic equation of state proposed by Estrin et al. ${ }^{6}$ The fluctuation field $H_{f}$ is defined by $H_{f}=S_{v} \ln \left(t_{m} / \tau_{0}\right)$, where $S_{v}$ is the coefficient of magnetic viscosity, related to the irreversible susceptibility and the magnetic viscosity as $S_{v}=S / \chi_{\text {irr }}$. The evaluation of $S$ and $\chi_{\text {irr }}$ is simultaneously done. The time dependence of the magnetization $\left[\mu_{0} M_{\text {irr }}(t)\right]$ was recorded for several applied demagnetizing fields ranging from $0.85 H_{\text {cr }}$ up to $1.15 H_{\text {cr }}$ at each selected temperature. All the demagnetization curves showed a time logarithmic dependence (Fig. 1) in the time range going from 500 to $1500 \mathrm{~s}$, where the magnetic viscosity $S$ $=d M / d$ Ln $t$ was evaluated. $\chi_{\text {irr }}$ is obtained from the slope of irreversible demagnetization curves $\mu_{0} M_{\text {irr }}$ versus the applied field (Fig. 2 and inset). The $\left(\mu_{0} M_{\text {irr }}, \mu_{0} H\right)$ values were determined from the $d \mu_{0} M / d t=c t$. condition in the $d \mu_{0} M / d t$ vs $\mu_{0} M$ curves: first the time derivative of the measured magnetization is plotted versus the magnetization $\mu_{0} M$ (as shown in the inset of Fig. 1), and then the intersection of a horizontal line $\left(d \mu_{0} M / d t=c t\right.$. $)$ with the different curves corresponding to the diverse fields applied in the measurements gives us the $\left(\mu_{0} M_{\text {irr }}, \mu_{0} H\right)$ values. In Fig. 3 we show the fluctuation field versus temperature for both

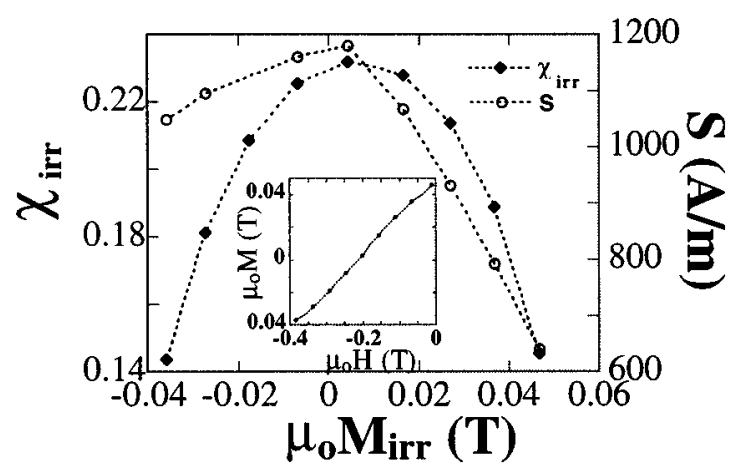

FIG. 2. $\chi_{\text {rr }}$ obtained from the slope of irreversible demagnetization curves $\mu_{0} M_{\text {irr }}$ vs the applied field (inset) and $S=d M / d \operatorname{Ln} t$ for S1 at $43 \mathrm{~K}$.

samples (as compared with the critical fields). The fact that the critical fields approximate to the fluctuation field at medium temperatures indicates the importance of thermal activation as a demagnetization mechanism at these temperatures. The $H_{f}$ values are significantly lower in S2 than in S1. Accepting the occurrence of a coherent rotation mechanism of demagnetization, the obtained $S_{v}$ values can be related to a so-called activation volume through $v_{a}=k_{B} T /\left(\mu_{0} M_{s} S_{v}\right)$, where $M_{S}$ is the saturation magnetization of the bulk material (about $4.77 \times 10^{5} \mathrm{~A} / \mathrm{m}$ ), and $v_{a}$ represents the volume of material involved in a single activation process. ${ }^{7}$ Commonly, $v_{a}$ is understood as the volume which is able to overcome the energy barrier when $H_{f}$ is applied (or, conversely, $H_{f}$ is the field at which the barrier for $v_{a}$ is equal to the thermal energy). In Fig. 4 we show the temperature dependence of the activation volume of both samples. A clear increase of $v_{a}$ with temperature is observed for the two samples. Taking into account that for both samples the fluctuation field scarcely changes with temperature, is clear that as we increase the temperature, the same field is able to reverse bigger volumes. Concerning $\mathrm{S} 1, v_{a}$ ranges within about one third and three times the mean particle volume. The particle size distribution as obtained from TEM observations indi-

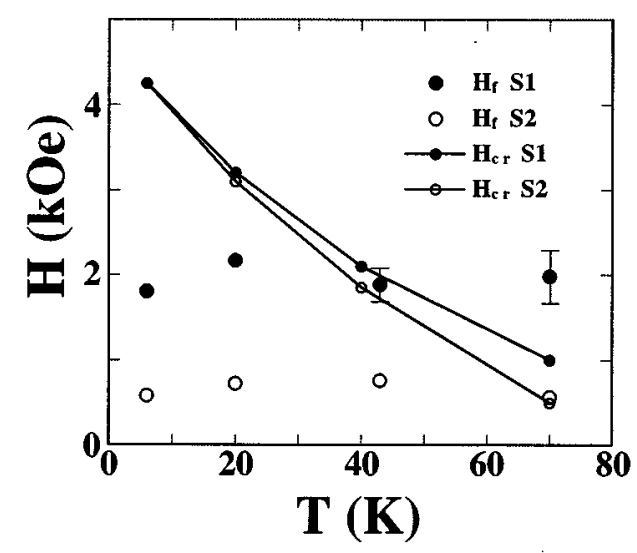

FIG. 3. Fluctuation field $\left(H_{f}=32 \cdot S_{v}\right)$ and critical field $\left(H_{\mathrm{cr}}\right)$ vs temperature for both samples. The error bars in $H_{f}$ come from the different field dependence of $\chi_{\text {irr }}$ and $S$. 


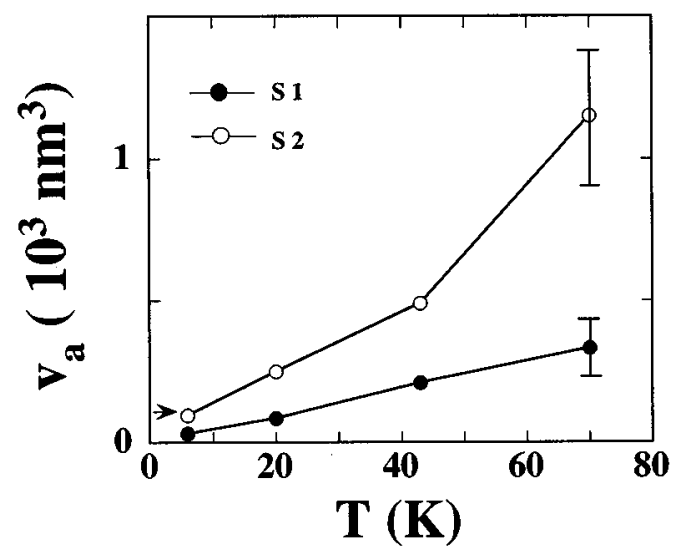

FIG. 4. Activation volume for $\mathrm{S} 1(\bullet)$ and $\mathrm{S} 2(\bigcirc)$. The arrow shows the mean particle volume for $\mathrm{S} 1$ obtained from TEM $\left(1.05 \times 10^{2} \mathrm{~nm}^{3}\right)$.

cates a non-negligible amount of particles with this largest volume. The activation volume is manifestly larger for S2, what indicates that demagnetizing interactions are more important in this sample that in S1. Only at the lowest temperatures is $v_{a}$ for $\mathrm{S} 2$ of the order of the particle volume obtained by TEM and x-ray diffraction (the arrow in Fig. 4) and at the highest temperature $v_{a}$ is about eleven times the mean particle volume (this volume is larger than the maximum volume detected by TEM), these suggests that the demagnetization process goes from an individual mechanism for S1 to a collective mechanism for S2 due to demagnetizing interactions.

The time dependence of the thermoremanence was also analyzed in terms of the $T \ln \left(t / \tau_{0}\right)$ scaling (see Ref. 8 for details). The scaling procedure consists on choosing the $\tau_{0}$ which makes all experimental relaxation curves corresponding to the different temperatures scale onto a single master curve that stands for the whole relaxation curve at the lowest temperature $\left(\tau_{0}=10^{-12} \mathrm{~s}\right.$ in the present case). Although the suitable $\tau_{0}$ is the same for the two samples, larger relaxation rates at low $T \ln \left(t / \tau_{0}\right)$ values and at very large $T \ln \left(t / \tau_{0}\right)$ values are observed for S2. The derivatives of the two experimental master curves, $\partial M / \partial\left[T \ln \left(t / \tau_{0}\right)\right]$ (which are proportional to the energy barrier distribution, ${ }^{8}$ evidence larger values of the density of the low energy barriers in S2 than in S1 (Fig. 5). We associate this to the enhancement of the demagnetizing interactions. The maximum of the energy barrier distribution is attained at similar energy values in both samples, as expected from the $H_{c}$ vs $T^{0.77}$ curves yielding similar anisotropy energy values. Considering the structure of the $f(E)$ vs $E / k_{B}$ curves the master curves were fitted using

$$
\begin{aligned}
M(t)= & M_{0} \int_{0}^{\infty} d E\left[p f_{1}(E)+(1-p) f_{2}(E)\right] \\
& \times \exp [-t / \tau(E)]
\end{aligned}
$$

corresponding to two log-normal distributions of energy barriers $f_{1}(E)$ and $f_{2}(E)$. The best fits of the experimental mas-

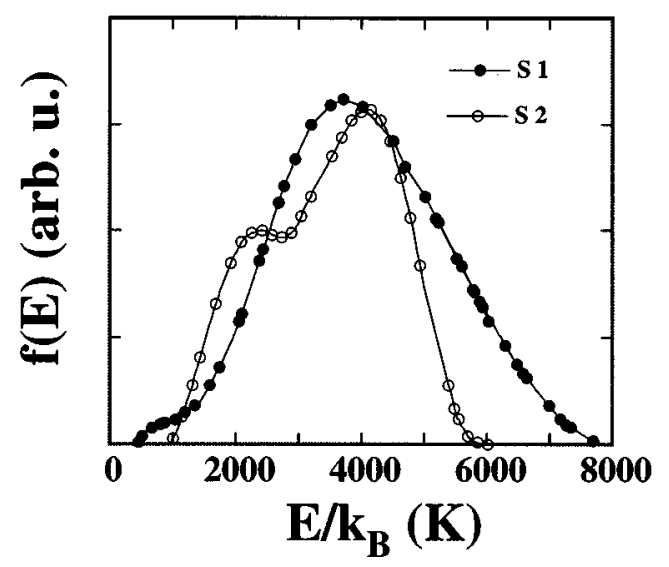

FIG. 5. Numerical derivative of the experimental master curves (magnetization vs $T \ln \left(t / \tau_{0}\right)$, with $\tau_{0}=10^{-12} \mathrm{~s}$ for 26 temperatures within 11 and 220 $\mathrm{K}$ with respect to the scaling variable for $\mathrm{S} 1(\mathbf{O})$ and $\mathrm{S} 2(\bigcirc)$.

ter curves to Eq. (1) are obtained with the following parameters: (a) S1: peaks of the distributions $T_{B 01}=1216 \mathrm{~K}$ and $T_{B 02}=3872 \mathrm{~K}$, widths $\sigma_{1}=0.74$ and $\sigma_{2}=0.40$, mixing parameter $p=0.19$ and (b) S2: $T_{B 01}=1664 \mathrm{~K}$ and $T_{B 02}=4352 \mathrm{~K}$, widths $\sigma_{1}=0.66$ and $\sigma_{2}=0.34$, mixing parameter $p=0.40$. $T_{B 01}$ describes the extra contribution of the lower energy barriers (demagnetizing interactions), while the $T_{B 02}$ is linked to the second contribution centered at higher energies and describes the contribution of noninteracting particles and/or particles with magnetizing interactions. The fit of the master curve in the case of S2 has been recorded up to $T \ln \left(t / \tau_{0}\right)=6800$, because above this value, it decays faster than a log-normal distribution function does. We note that if the amount of low-energy barriers is enhanced in S2 with respect to $\mathrm{S} 1$, due to larger interparticle interaction, it is then reasonable that a decrease in the amount of the large energy barrier is also observed. The second energy distribution $f_{2}(E)$ is comparable to the blocking temperature distribution $F\left(T_{B}\right)$ derived for $\mathrm{S} 1$ from thermoremanent data and to the volume distribution obtained from the fitting of the high temperature $M(H)$ curves to a distribution of Langevin functions, ${ }^{1}$ since in this sample the overall magnetic behavior is dominated by $f_{2}(E)$ ( $p$ is only 0.19 ).

This work has been partially supported by the catalan government through Grant No. GRQ 1012, and the Spanish CICYT through Grant No. MAT94-1024-C02-02.

${ }^{1}$ X. Batlle, M. García del Muro, A. Labarta, and P. Görnert, J. Magn. Magn. Mater. 157-158, 191 (1996); Phys. Rev. B (to be published).

${ }^{2}$ T. Ido, O. Kubo, and H. Yokoyama, IEEE Trans. Magn. MAG-22, 704 (1986).

${ }^{3}$ P. Görnert, E. Sinn, and M. Rösler, Key Eng. Mater. 58, 129 (1991).

${ }^{4}$ H. Pfeiffer, and W. Schüppel, Phys. Status Solidi A 119, 259 (1990).

${ }^{5}$ D. Givord, P. Tenaud, and T. Viadieu, J. Magn. Magn. Mater. 72, 247 (1988).

${ }^{6}$ Y. Estrin, P. G. McCormick, and R. Street, J. Phys., Condens. Matter 1, 4845 (1989).

${ }^{7}$ E. P. Wohlfarth, J. Phys. F 14, L155 (1984).

${ }^{8}$ A. Labarta, O. Iglesias, Ll. Balcells, and F. Badia, Phys. Rev. B 48, 10240 (1993); O. Iglesias, F. Badia, A. Labarta, and Ll. Balcells, J. Magn. Magn. Mater. 140-144, 399 (1995). 